\title{
A Comparative Study of Ultrasonogram and Computed Tomography in Cases of Blunt Abdominal Trauma
}

\author{
Uma Maheswara Reddy V ${ }^{1}$, Ravi M Godavarthi ${ }^{2}$, Ramakrishna Baru ${ }^{3}$, Sunitha ${ }^{4}$, Gowtham Surya Duddu \\ ${ }^{1}$ Assistant professor, Department of Radiology, ${ }^{2}$ Associate professor, Department of Radiology, ${ }^{3}$ Professor. Department \\ of Radiology, ${ }^{4}$ Professor Department of Radiology, ${ }^{5}$ Resident, Department of Radiology, Narayana Medical College, \\ Chinthareddypalem, Nellore, Andhra Pradesh, India
}

Corresponding author: Dr. Ravi M Godavarthi, Associate professor, Department of Radiology, Narayana Medical College, Chinthareddypalem, Nellore, Andhra Pradesh, India

DOI: $10.21276 /$ ijcmsr.2018.3.2.36

How to cite this article: Uma Maheswara Reddy V, Ravi M Godavarthi, Ramakrishna Baru, Sunitha, Gowtham Surya Duddu. A comparative study of ultrasonogram and computed tomography in cases of blunt abdominal trauma. International Journal of Contemporary Medicine Surgery and Radiology. 2018;3(2):B152-B155.

\section{A B S T R A C T}

Introduction: Trauma accounts for one of the reason for majority of deaths in people younger than 45 years of age and is also a preventable cause of death. Ultra sonogram (US) of the abdomen appears to be a useful and important tool in diagnosis of any kind of injury to the abdominal organs due to BUT. Our aim of the present study was to assess the role of Ultrasonography in patients with BAT and record the findings and to perform CT in cases negative for US.

Material and methods: A prospective study for 12 months was conducted by the department of Radiology among cases of Blunt abdominal trauma. Hemodynamically stable patients were performed Ultra sonogram, and computed tomography in cases inconclusive by US and in cases highly suspicious of abdominal injury. The statistical analysis was performed by using SPSS (Version 1.0).Chi-Square test was done to find the significant difference and ' $p$ ' value $<0.05$ was considered significant. Results: 548 patients with 368 males and 180 females, mean age of 34.42 \pm .4 years were included. The average Injury severity score (ISS) was $17.9 \pm 1.0$ (Mean \pm SD). US alone in $84.3 \%$ of cases and both US and CT in $15.7 \%$ of cases were done. 58 cases had US score $<3$ and 490 cases had US score $>3$. US had a sensitivity of $94 \%$, specificity of $100 \%$ and accuracy of $97 \%$ in detection of free fluid in peritoneum. Liver was the most common organ injured and observed in 348 cases (63.5\%). Conclusion: Our study strongly suggests that CT scans should be followed by imperative US scans or in cases which are negative by US but clinically strongly suspicious of organ injury or damage. However accurate imaging diagnosis and hemodynamical stability are the main determinants that determine the strategy of management of cases of BAT.

Key words: Blunt abdominal Trauma, Ultrasonogram, Computed tomography, sensitivity.

\section{INTRODUCTION}

Trauma accounts for one of the reason for majority of deaths in people younger than 45 years of age and is also a preventable cause of death. The loss of years of life due to trauma is more than combined years of life loss of malignancy, heart disease and stroke combined. The energy impacted during a blunt trauma is distributed over a wide area compared with penetrating injuries and the forces involved during the impact create both shear and tensile strain. Examples for these include falls, automobile and motor vehicle accidents and sports related injuries. ${ }^{1}$ Pain, subtle physical signs, and masked by intoxication and head injury appears to be main reasons in case of missing abdominal injuries in cases of blunt abdominal trauma. Clinical diagnosis in blunt injuries is a challenging task to the surgeon or physician due to lack of specific findings in these high velocity injuries. ${ }^{2}$ In addition presence of associated head, thoracic injuries may divert the physician to miss the observation of intra abdominal injury. The likelihood of injury to an individual organ depends upon the impact of velocity and mechanism of trauma and also the vulnerability of the patient at the time of the event. In most of the literature reports, liver and spleen are the most common organs affected. Other organ which may be at risk includes kidneys, bowel, mesentery, pancreas, adrenals, and diaphragm and intra abdominal vessels. Neurological impairment due to traumatic event itself may limit in arriving a diagnosis by clinical examination. Hemodynamically stable patients and patients who respond to initial management often require further diagnostic evaluation. ${ }^{3}$ In most of the situations of BUT, initially diagnostic peritoneal lavage was a choice of procedure in suspected cases of hemoperitoneum. But DPL has a lot of limitations and unnecessary lavage may be done and not indicated during pregnancy. Ultra sonogram (US) of the abdomen appears to be a useful and important tool in diagnosis of any kind of injury to the abdominal organs due to BUT. But an important hindrance factor previously was resolution, but with modern advances and Focussed assessment with sonography and trauma [FAST] the choice of primary investigation in BAT has shifted from DPL to US. However the even improved US have only 50\% specificity in solid organ injuries. ${ }^{4}$ Computed tomography [CT] has 
become the choice in advanced trauma centres as a primary modality of investigation in cases with BUT. CT evaluates retro peritoneum and abdomen with an additional advantage in also assessing the functional status of organs and skeletal injuries. Most of the reports and findings from various studies demonstrated CT as a better diagnostic choice than US in cases of BAT. Our aim of the present study was to assess the role of Ultrasonography in patients with BAT and record the findings and to perform CT in cases negative for US.

\section{MATERIAL AND METHODS}

A prospective study was conducted at Department of Radiology in association with Department of surgery and Emergency Medicine for a period of twelve months from January 2106 to December 2016. All the patients attending the emergency medicine with history of Blunt Abdominal Trauma (BAT) due to any cause and referred from emergency and surgery for imaging studies (Ultra sonogram or computed tomography) of abdomen were included in the study. Cases that were hemodynamically unstable, with obvious abdominal injuries or penetrating injuries were directly taken to operational theatre and excluded from the study. The study was approved by the institutional ethical committee and guidelines of the committee were followed in the study. The study was explained to all the cases or relatives and consent was obtained. Patients with only pain abdomen were excluded from the study. The socio demographic features, history of trauma, mechanism of injury were noted and entered in a separate predesigned questionnaire form. Children $<14$ years were excluded from the study.

Patients were transported to the department of radiology and performed both ultra sonogram of abdomen and CT of abdomen and pelvis. Ultra sonogram and CT when necessary were performed by a single senior radiologist with an experience of minimum 5 years for all the cases to avoid bias reporting. US examinations were performed by SSA270 Toshiba, Japan device with a $3.75 \mathrm{MHz}$ probe device. Presence of free fluid in the abdominal cavity was accepted as a sign of hemoperitoneum. Nine important anatomic areas were evaluated thoroughly which included bilateral subphrenic spaces, Sub hepatic space, and perisplenic area, free edge of liver, splenic tip, bilateral paracolic gutters and pelvis.

CT examination: This was done in cases with findings inconclusive on ultra sonogram, clinical examination positive for injury but negative on ultra sonogram and in cases of hemoperitoneum to localise the site of bleed. The findings of ultra sonogram were compared with CT.

\section{STATISTICAL ANALYSIS}

The statistical analysis was performed by using SPSS (Version 1.0).Chi-Square test was done to find the significant difference and 'p' value $<0.05$ was considered significant.

\section{RESULTS}

A total of 548 patients (368 males and 180 females) with blunt abdominal injury referred from Emergency and Department of General surgery were enrolled in the study. The male to female ratio in the study was $2.04: 1$. The mean

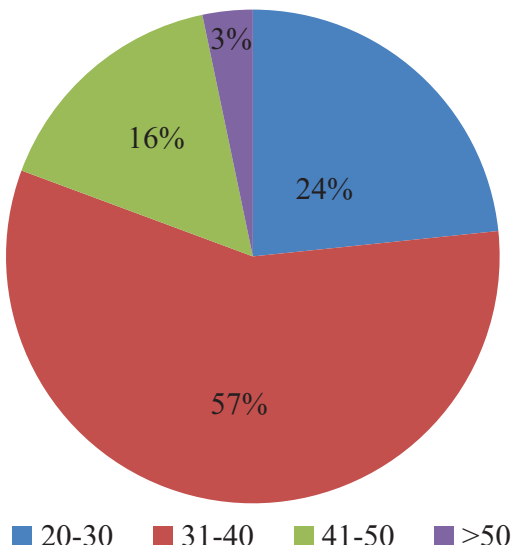

Figure-1: Age wise distribution of cases in the study (Years)

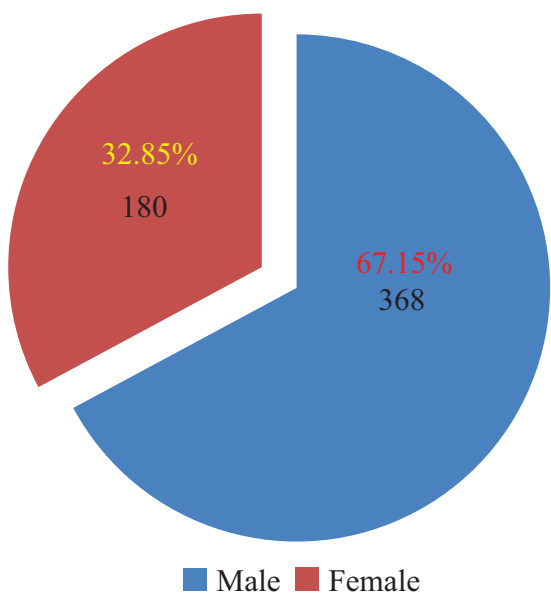

Figure-2: Gender Distribution of cases in study

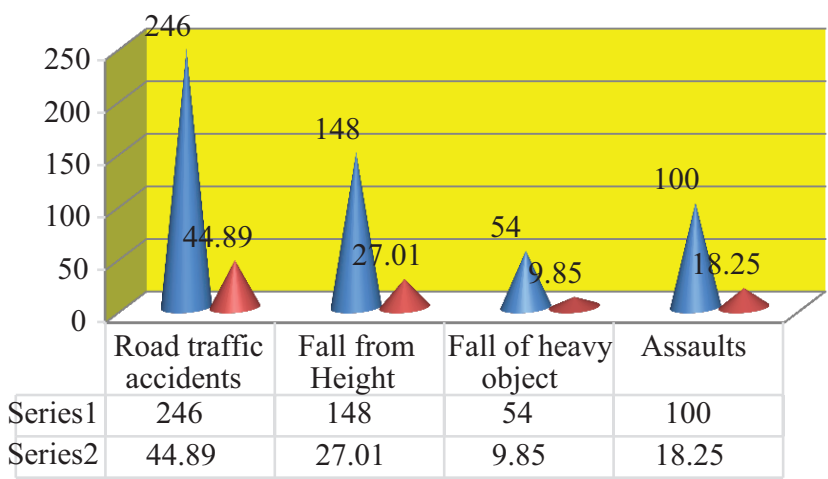

Figure-3: Type of Injury among the cases in the study

\begin{tabular}{|l|c|c|}
\hline Signs and Symptoms: & Number & $\%$ \\
\hline Abdominal pain & 548 & 100 \\
\hline Nausea & 477 & 87 \\
\hline Vomiting & 477 & 87 \\
\hline Abdominal fullness & 477 & 87 \\
\hline Tenderness & 548 & 100 \\
\hline Guarding & 417 & 76 \\
\hline Rebound tenderness & 460 & 84 \\
\hline Systolic BP<100mmhg & 307 & 56 \\
\hline Average Injury severity score (Mean \pm SD) & \multicolumn{2}{|c|}{$17.9 \pm 1.0$} \\
\hline Both US and CT & 86 & 15.7 \\
\hline
\end{tabular}

Table-1: Distribution of clinical signs and symptoms among the cases in the study 


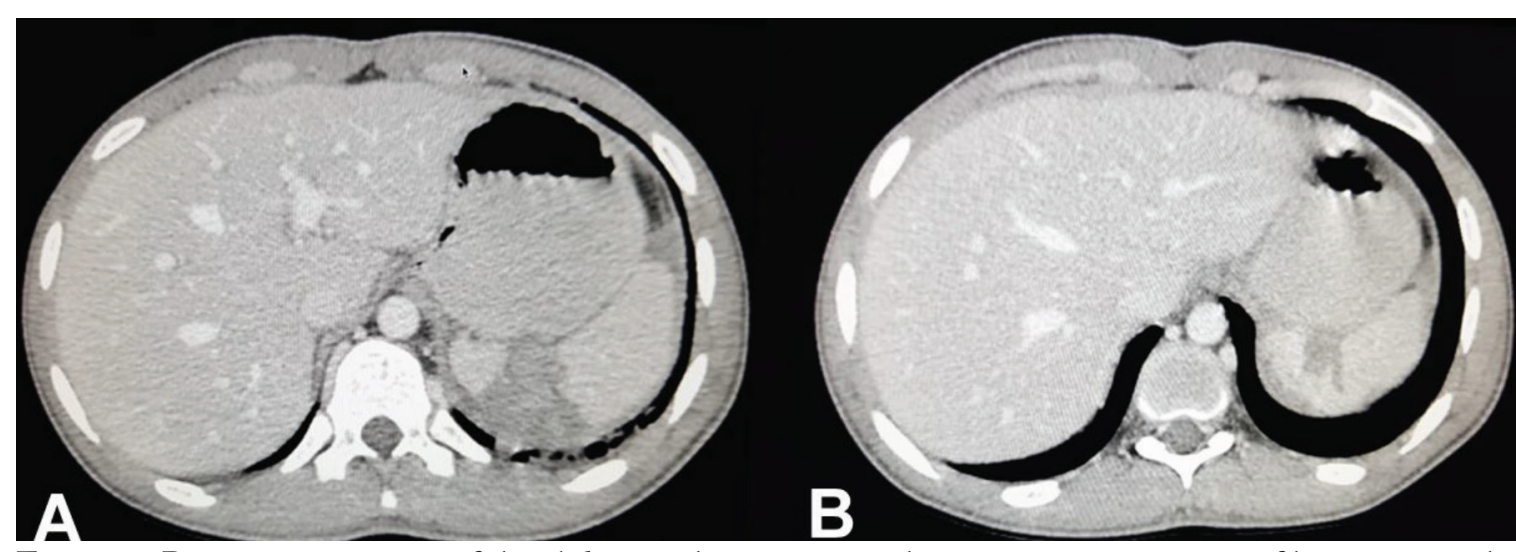

Figure-4: Portovenous images of the abdomen showing non enhancing areas suggestive of laceration in the spleen superior pole. Minimal left pleural effusion is also noted.

\begin{tabular}{|l|c|c|}
\hline Organ & No & \% \\
\hline Liver & 348 & 63.50 \\
\hline Spleen & 248 & 45.26 \\
\hline Kidneys & 24 & 4.38 \\
\hline Pancreas & 2 & 0.36 \\
\hline Bowel and Mesentery & 12 & 2.19 \\
\hline Urinary Bladder & 2 & 0.36 \\
\hline \multicolumn{2}{|c|}{ Table-2: Distribution of Organ injury among the cases in the } \\
study \\
\hline
\end{tabular}

age of the participants in the study was $34.42 \pm 8.4$ years (range 21 to 58 years) and maximum participants were in the age group of $31-40$ years (57.30\%) followed by $20-30$ years (23.36\%). [Figure-1 and 2] Road traffic accidents were the most common cause of BUT in the study (44.89\%) followed by fall from height (27.01\%), assaults (18.25\%) and fall of heavy object (9.85\%). [Figure-3]The average Injury severity score (ISS) was $17.9 \pm 1.0($ Mean \pm SD). Among the clinical symptoms, abdominal pain was the most common seen in $100 \%$ of cases followed by nausea, vomiting, and abdominal fullness in $87 \%$ of cases. Tenderness was elicited in $100 \%$ cases, rebound tenderness in $84 \%$, guarding in $76 \%$ and absence of bowel sounds in $56 \%$ of cases. Among all the cases, $94 \%$ of cases had BP $>100 \mathrm{~mm}$ of $\mathrm{Hg}$. [Table-1]

In our study US and CT, both were done in 86 cases (15.7\%) and US alone was performed in 462 cases (84.3\%). In US free fluid with attenuation value $>30$ Hounsfield Units was labelled as hemoperitoneum. Scoring of hemoperitoneum by US findings was done as per the scoring system of Huang et al. ${ }^{5}$ In our study, 58 cases had US score $<3$ and 490 cases had US score $>3$. In CT study, scoring of hemoperitoneum was done as per the guidelines of Federle and Jeffrey as small, moderate and large. By CT, 28 cases were scored as small, 214 as moderate and 306 as large. All the cases scored as small by CT were managed conservatively and followed. All the moderate and large were surgically managed by Laparotomy. In our study, US had a sensitivity of $94 \%$, specificity of $100 \%$ and accuracy of $97 \%$ in detection of free fluid in peritoneum.

Organ Injuries: In the present study, liver was the most common organ injured and observed in 348 cases (63.5\%), followed in order by Spleen 248 cases (45.26\%), kidneys 24 cases (4.38\%), Bowel and mesentery 12 cases $(2.19 \%)$,

pancreas 2 cases $(0.36 \%)$ and urinary bladder in 2 cases (0.36\%). 28 cases had multi organ injuries. [Table-2] Out of 548 cases in the study, CT and US reported similar findings in 428 cases and in 28 cases additional findings were noted but management of the cases did not differ with the additional findings in CT. [Figure-4]

In 548 cases US and CT demonstrated, either hemoperitoneum or organ injury or both. In our study US had a sensitivity of $94 \%$, specificity of $100 \%$. CT findings in our study detected all the cases with hemoperitoneum or parenchymal injury or both and had sensitivity, specificity and accuracy of $100 \%$ when compared with US. Hence in our study CT had a definite advantage than US in visualization of minor splenic and kidney injuries and rib fractures, lung contusions.

\section{DISCUSSION}

BAT accounts for majority of cases appearing for US and $\mathrm{CT}$ in a trauma care and tertiary care hospital. A precise diagnosis in cases of BUT obviates the need for unnecessary Laparotomy and complications, hence arriving a definite diagnosis by imaging studies may help the surgeon in this regard. In the present study, Road traffic accidents accounts for majority of cause of BAT in our study (44.89\%) as reported in the findings of Mohapatra et al, ${ }^{6}$ who reported the incidence of RTA as $54 \%$ and Kulakarni et al who reported as $64 \%$ which is higher than our study. ${ }^{7}$ In our study, BAT was most common in the age group of 31-40 years which is observed in the literature reports of many studies universally and also in India. This age is the highly vulnerable group as they are more actively involved in works externally and also in motor traffic accidents. ${ }^{8}$ Abdominal pain was the most common symptom in our study as also reported by Farahmand et al in his study. ${ }^{9}$ In our study, tenderness was the most common clinical sign with other signs like guarding, rebound tenderness. However few studies reported that guarding, Hypotension and rebound tenderness were the common findings in cases of BAT. This is explained by the reason that signs depend upon the type of injury and shear tensile strength which caused the injury and type of injury which can be organ parenchymal damage, hemoperitoneum or major organ injury.

In our study among 58 cases with US score <3, 53 were 
managed conservatively and 3 cases were operated for bladder rupture and 2 for retroperitoneal hematoma and rest 490 cases required operative management depending upon the imaging result and clinical status of the patient. Findings of our study were in comparison with findings of Mallik et al who reported the same in his study, but were contrast to the findings that few cases with US score $>3$ were managed conservatively. ${ }^{10}$ Hence our study strongly supports that hemodynamic stability is prime factor in management of cases of BAT. Many of the studies earlier reported that presence of hemoperitoneum mandates laparotomy. But in our study we observed that 8 cases identified as small in CT were managed conservatively which is consistent with the recent trend in management of most of the solid viscous injuries. However in all the cases with moderate and large in CT imaging management was by laparotomy only and none of the cases were conservatively managed. This finding of our study is consistent with the findings of Kane $\mathrm{M}$ et al who reported that $98 \%$ of cases with moderate and large by CT imaging were surgically managed by laparotomy. ${ }^{11}$ In our study the accuracy of US in detection of hemoperitoneum was $97 \%$ which is similar to findings of Padhani et al who reported 100\% accuracy in his study with both US and CT evaluation of BAT. ${ }^{12}$ This clearly states that US is reasonably accurate in detection of hemoperitoneum which is a frequent pointer in presence of abdominal injury

As majority of the studies point in their findings, that liver is the major organ of injury which is on par observed in our study also. Spleen was the $2^{\text {nd }}$ major organ followed by liver and all the cases with major organ damage were managed by surgical intervention depending upon the grading of injury. Majority of the major organ injuries were picked by US except in cases with few cases of splenic injury, and urinary bladder rupture, retroperitoneal haematomas which were detected by CT, in these cases the sensitivity was low and was high by CT which is on par with findings of Tripathi et al. ${ }^{13}$ The accuracy of US in predicting multiple organ injuries was $96 \%$ but in CT it was $100 \%$ and PPV was $96 \%$ by US and $100 \%$ by CT. In detecting splenic injury, mesenteric injuries and kidney injuries the sensitivity and PPV of CT was higher than US and similar findings were reported by Richards JR et al in their study. ${ }^{14}$

Conclusion

To conclude, from our study CT is always a superior diagnostic modality than US in cases of organ injury in BAT. However US can be performed as a initial imaging modality in all the cases of BAT and however US can miss few cases of minor organ parenchymal damage and retroperitoneal hematomas masked by dilated loops or thickened bowel walls. Hence it is imperative that all cases of BAT are to be followed by CT after US. Our study strongly suggests that CT scans should be followed by imperative US scans or in cases which are negative by US but clinically strongly suspicious of organ injury or damage. However accurate imaging diagnosis and hemodynamical stability are the main determinants that determine the strategy of management of cases of BAT. US is a sensitive investigation in diagnosing cases of hemoperitoneum than organ specific damages and $\mathrm{CT}$ is a better diagnostic modality in organ damage than
US and CT however has less sensitivity in detection of mesenteric tears and small bowel injury.

\section{REFERENCES}

1. Leung E, Wong L, Taylor J. Non-operative management for blunt splenic trauma in children: An updated literature review. Surgical Practive. 2007;11(1):29-35.

2. National Trauma Data Bank Report 2005. Am Coll Surg 1-60, 2005.

3. Tien HC, Tremblay LN, Rizoli SB, Gelberg J, Spencer F, Caldwell C, Brenneman FD. Radiation exposure from diagnostic imaging in severely injured trauma patients. J Trauma. 2007;62(1):151Y156.

4. Richards JR, Schleper NH, Woo BD, Bohnen PA, McGahan JP. Sonographic assessment of blunt abdominal trauma: a 4-year prospective study. J Clin Ultrasound 2002; 30 (2):59-67.

5. Huang MS, Liu ML, Wu JK, et al. Ultrasonography for the evaluation of hemoperitoneum during resuscitation; a simple scoring system. J Trauma 1994; 36: 173- 177.

6. Mohapatra S, Pattanayak SP, Rao KRRM, Bastia B. Options in management of solid visceral injuries from blunt abdominal trauma. Indian J Surg. 2003;65 (3):263-8.

7. Kulkarni MS, Hindlekar MM. Primary repair or colostomy in the management of civilian colonic trauma. Indian J Gastroenterol. 1995;14 (4):54-6.

8. Salim A, Sangthong B, Martin M, Brown C, Plurad $\mathrm{D}$, Demetriades D.Whole body imaging in blunt multisystem trauma patients without obvious signs of injury: results of a prospective study. Arch Surg. 2006;141(5): 468-473.

9. Farahmand N, Sirlin CB, Brown MA, Shragg GP, Fortlage D, Hoyt DB, Casola G. Hypotensive patients with blunt abdominal trauma: performance of screening US. Radiology. 2005;235(2):436Y443.

10. Mallik K, Vashisht S, Thakur S, Srivastava D N. Comparative evaluation of ultrasonography and CT in patients with abdominal trauma: A prospective study. Indian J Radiol Imaging 2000;10 (5):237-243.

11. Kane M.; Dorfman; Kronan. Et al Efficacy of CT following peritoneal lavage in abdominal trauma. Journal of computed tomography.1987;11 (6): 998-1002.

12. Padhani et al Computed tomography in blunt abdominal trauma audit of usage and image quality. British Journal of Radiology. 1992; 65 (7): 397-402.

13. Tripathi MD, Srivastava RD, Nagar AM, Pratap VK, Dwivedi SC. Blunt abdominal trama with special reference to early detection of visceral injuries. Indian I Surg. 1991;53 (1):179-84.

14. Richards JR, Schleper NH, Woo BD, Bohnen PA, McGahan JP. Sonographic assessment of blunt abdominal trauma: a 4-year prospective study. J Clin Ultrasound 2002; 30 (5):59-67.

\section{Source of Support: Nil; Conflict of Interest: None}

Submitted: 15-05-2018; Accepted: 23-06-2018; Published online: 26-06-2018 